\title{
Phénologie et variations du dermecos chez quelques espèces de Spinturnicidae (Acarina, Mesostigmata)
}

\author{
par J. DEUNFF * et J.-C. BEAUCOURNU * \\ * Laboratoire de Parasitologie (Entomologie médicale), Faculté de Médecine, \\ avenue du Professeur Léon-Bernard, F 35043 Rennes Cedex.
}

RESUME. La mise au point de fiches de terrain a permis de réaliser la première étude exclusivement consacrée à la phénologie et au dermecos des Spinturnicidae.

Phénologie.

Les espèces étudiées appartenant aux genres Spinturnix et Eyndhovenia ont une reproduction et un maximum de population en été. L'hiver, seuls quelques adultes constituent les stases de résistance.

Dans l'espèce Paraperiglischrus rhinolophinus, au contraire, le maximum de population et la reproduction ont lieu en hiver.

Dermecos.

Les espèces du genre Spinturnix sont localisées toute l'année au niveau du patagium des chiroptères. Nous retrouvons cette localisation chez E. euryalis mais uniquement en été car l'hiver les quelques adultes résistants se réfugient dans le pelage bordant le patagium.

Chez $P$. rhinolophinus les mâles vivent sur le patagium, les femelles et les nymphes presque exclusivement sur l'uropatagium dorsal.

Phenology and variations of dermecos in some species of Spinturnicidae (Acarina, Mesostigmata).

SUMMARY. Original cards allow to make the first study exclusively on phenology and dermecos of Spinturnicidae.

Phenology.

Species studied of the genera Spinturnix and Eyndhovenia have reproduction and maximum population during summer : In winter some males and females are resistant stages.

Accepté le 19 août 1980 .

Annales de Parasitologie humaine et comparée (Paris), t. 56, $\mathrm{n}^{\circ} 2$. 
On the contrary Paraperiglischrus rhinolophinus is a winter species.

Dermecos.

Species of the genus Spinturnix are found on the wing membranes of bats all the year. E. euryalis is located on the wing-membrane in summer and in the coat bordering on this one during winter.

Males of P. rhinolophinus are on the wing membranes, females and nymphs on back tail membrane.

\section{Introduction}

Dans une précédente publication (Deunff, 1977), nous avions limité notre étude à la systématique des Spinturnicidae de la région paléarctique occidentale. Cette étape était indispensable, en préalable à une approche écologique de ces acariens.

Les publications concernant cette famille sont très nombreuses mais presque toutes consacrées à la morphologie et à la systématique. Les difficultés de l'observation in vivo de ces acariens et la taxonomie encore peu satisfaisante expliquent l'orientation prise jusqu'à ce jour par les recherches. Nous devons toutefois signaler l'étude de Dusbabek (1972), sur l'écologie des acariens de chauves-souris d'Europe centrale, dans laquelle les Spinturnicidae sont mentionnés; Herrin (1975) a noté la fréquence du parasitisme des chiroptères par les Spinturnicidae au Venezuela; Reisen et al. (1976) ont étudié l'écologie hivernale des ectoparasites de Myotis velifer dans l'Oklahoma (U.S.A.) ; enfin, Haitlinger (1978) a publié une étude sur les Spinturnicidae, Argasidae et Ixodidae des chauves-souris de Silésie en automne-hiver.

Après deux années d'études sur le terrain, nous donnons nos premières observations écologiques sur ces populations d'ectoparasites. A notre connaissance, cette étude est la seule consacrée exolusivement à l'écologie de cette famille (1).

Nous tenons à remercier $\mathrm{M}^{\text {Hes }}$ Bach-Hamba et Henri, MM. Launay, Omais et Menguy qui nous ont aidés dans la récolte de notre matériel d'étude.

\section{Matériel et méthode}

\section{1. - Techniques de terrain.}

D'octobre 1977 à septembre 1979, nous avons suivi quelques populations de chauves-souris dans l'Ouest de la France afin d'étudier leurs ectoparasites. L'intervalle de temps séparant nos visites dans les grottes l'hiver, puis les greniers et les clochers

(1) Une partie de cette étude a fait l'objet d'un mémoire de Diplôme d'études approfondies d'Ecologie-Ethologie à la Faculté des Sciences de Rennes (J. Deunff, 1978). 
l'été, a varié de 15 jours à 2 mois selon la fréquentation des gîtes par les chiroptères afin d'éviter d'en perturber les populations.

Dans l'intention de codifier les observations, nous avons mis au point des fiches de terrain d'un format $21 \times 15 \mathrm{~cm}$ en bristol. Ces fiches rigides sont de deux sortes : - fiche biotope: une fiche par visite, rassemblant les caractéristiques générales du site : type (grotte, carrière, toiture...), date, localité, faune et flore associées, température, humidité, plan,

- fiche chiroptère-ectoparasite : une fiche par chiroptère parasité, portant les renseignements concernant l'hôte: espèce, sexe, âge physiologique, numéro de capture ; ectoparasites autres que les Spinturnicidae. La localisation précise des Spinturnicidae sur l'hôte figure également sur cette fiche : espaces interdigitaux, face dorsale ou ventrale du patagium (2) droit ou gauche, uropatagium dorsal ou ventral. Ces renseignements peuvent être portés très rapidement sur des schémas dorsaux et ventraux des membranes imprimés sur la fiche. Le seul code à utiliser concerne le côté du patagium : droit (x) ou gauche (o) (fig. 1).

Ces fiches sont ensuite exploitées au laboratoire.

Lors de nos récoltes nous avons tenté de garder en survie nos acariens au moyen de flacons de verre dont le fond est garni de plâtre ou d'une éponge humide. Les Spinturnicidae de chaque chauve-souris sont placés dans un tube bouché par du coton cardé, puis déposés dans ce «flacon de survie». Cette technique ne permet qu'un à deux jours de survie pour la plupart des espèces.

Les mesures physiques dans les gîtes ont été prises par un psychromètre « Richard » pour l'humidité et un thermomètre à sonde à lecture digitale " Groux » pour la température.

\section{Techniques de laboratoire.}

L'étude du cycle complet et du comportement des Spinturnicidae exige tout d'abord la maîtrise des élevages de leurs hôtes. L'élevage des chiroptères en laboratoire est possible, citons par exemple, les travaux de Belashova (1964), Davis et Luckens (1966), Tesh et Arata (1967), Wimsatt (1970). Malheureusement, ne disposant pas de l'équipement nécessaire à une telle expérimentation, nous n'avons pu qu'obtenir la survie de ces animaux pendant la période d'hibernation, dans une pièce à température constante $\left(\mathrm{T}^{\circ}=10^{\circ} \mathrm{C}\right.$, humidité $\left.100 \%\right)$.

\section{Biotopes.}

L'étude des biotopes concerne plus spécialement les chiroptères hôtes, mais comme nous le verrons ultérieurement, les caractéristiques des gîtes influent également sur les parasites.

(2) Patagium: membrane alaire; uropatagium: membrane réunissant les membres postérieurs et englobant la queue chez les espèces étudiées ici. 
206

J. DEUNFF ET J.C. BEAUCOURNU

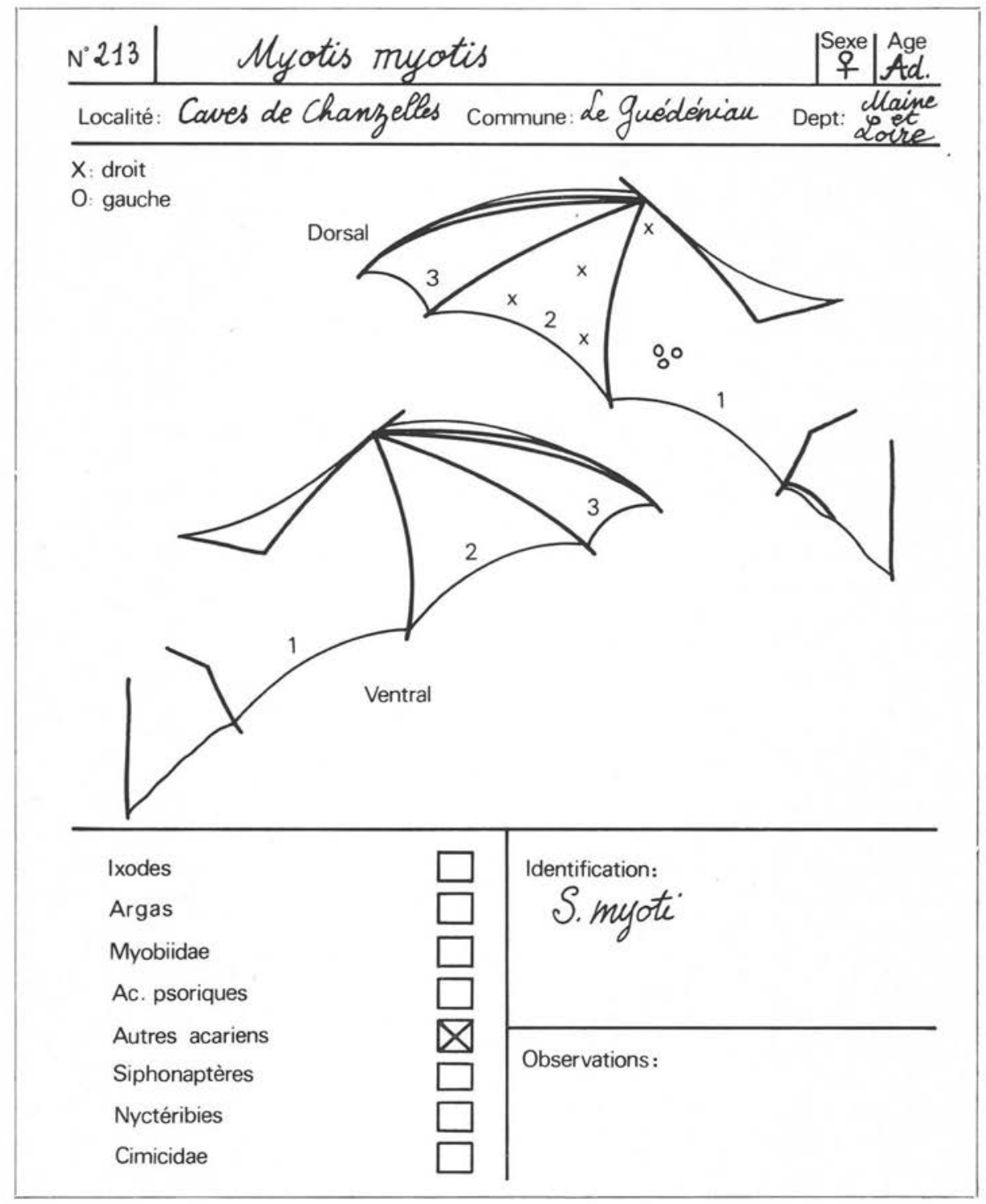

Fig. 1. Fiche de terrain "chiroptère-ectoparasite». 
Deux types de gîtes ont été étudiés :

\section{Stations hypogées.}

Constituées par des carrières souterraines, toutes situées en Maine-et-Loire (Le Guédéniau, Cornillé-les-Caves, St-Georges-des-Bois), creusées, à partir du $\mathrm{xv}^{\mathrm{e}}$ siècle (Fraysse J. et Fraysse C., 1963), dans le calcaire crétacé pour l'extraction de la pierre à bâtir.

Plusieurs types de microclimats se sont établis depuis les zones d'entrée très dégradées à luminosité peu atténuée, jusqu'aux cavités profondes, totalement sombres et thermiquement stables. Toutefois, ces derniers gîtes ne sont, pratiquement, fréquentés qu'en hiver.

En fonction des microclimats, on trouve une faune variée occupant tous les ans, dans la majorité des cas, les mêmes fissures ou les mêmes voûtes pour une espèce donnée (Myotis mystacinus (3), M. bechsteini, M. emarginatus). Quelques tas de guano, très abondants, sont les témoins des importantes colonies anciennes, Rhinolophus ferrumequinum essentiellement. Certaines carrières (caves de Chanzelles, commune du Guédéniau) ont été partiellement transformées en village troglodyte à certaines époques. On retrouve alors des vestiges de murs de pierre constituant à présent des gîtes pour certaines chauves-souris (Eptesicus serotinus, Pipistrellus pipistrellus, Plecotus auritus, P. austriacus).

L'hiver, quelques collections d'eau peuvent se former et recouvrir une partie $\mathrm{du}$ sol. Les températures hivernales sont de l'ordre de $8^{\circ}$ à $12^{\circ}$ en moyenne selon les endroits et l'humidité est de $100 \%$. La stabilité de l'air est variable avec l'orientation des galeries et leur distance de l'entrée. Ce dernier facteur est déterminant en ce qui concerne la présence de certaines colonies ( $R$. ferrumequinum).

\section{Stations épigées.}

L'été nous avons suivi les colonies dans deux de leurs gîtes estivaux : le grenier du château de Durtal et le clocher de l'église de Blou (tous les deux dans le Maineet-Loire) où l'on trouve presque exclusivement des femelles et leurs jeunes.

Ces deux stations présentent un à trois étages de poutres surmontés de toitures, sous les ardoises desquelles les chauves-souris se tiennent groupées en essaims. A cette saison, la température à ce niveau, dépasse largement $30^{\circ}$. Sur le plancher où vient s'accumuler le guano, les températures relevées varient de $21^{\circ}$ à $24^{\circ}$.

L'éclairement est atténué, mais reste important.

\section{Influence des facteurs abiotiques et biotiques.}

Si l'on étudie les rapports étroits existant entre les parasites et leurs hôtes, on peut penser que les Spinturnicidae, vivant en "milieu tamponné " sur leur hôte,

(3) Qui représente peut-être un complexe dans la région considérée. Myotts brandti semble toutefois absent. 
sont peu concernés par les facteurs abiotiques. Mais ce serait méconnaître la biologie des chiroptères dont la température centrale varie considérablement en fonction des saisons. Durant l'hibernation, cette température est peu différente de la température extérieure, ainsi un Myotis sp. placé dans un milieu à $5^{\circ} \mathrm{C}$ avait une température centrale de 5,8 (in Grassé, 1955). Nous pouvons, de même, citer une Barbastelle en hibernation, littéralement enchassée sur 3 côtés par des coulées de glace. En fait, les Spinturnicidae sont donc soumis aux mêmes variations de température que leurs hôtes, et nous verrons qu'ils doivent soit les subir, soit modifier leur comportement.

Aucune étude n'a, jusqu'à présent, été faite en ce qui concerne le système hormonal et le métabolisme des hormones chez ces acariens. Il est cependant possible de penser, étant donné leur régime hématophage, que les hormones de l'hôte jouent un rôle dans le cycle de reproduction des parasites. Nous avons, en effet, constaté que les parturitions chez les Spinturnicidae ont lieu presque à la même époque que celles des hôtes. Un tel phénomène est bien connu chez certaines puces de lapin, Spilopsyllus et Cediopsylla, dont le cycle de reproduction est lié au cycle hormonal de l'hôte (Rothschild et Ford, 1973). Toutefois, les facteurs abiotiques tels que température et humidité jouent peut-être ce rôle de catalyseur chez nos acariens.

\section{Etude écologique.}

Actuellement, peu de renseignements concernant l'écologie des Spinturnicidae figurent dans la littérature scientifique mondiale. Quelques données concernent la localisation de ces parasites sur leurs hôtes: le patagium constitue presque exclusivement le dermecos (4) signalé par les auteurs ainsi que l'uropatagium, sans qu'une localisation précise soit donnée comme une caractéristique d'espèce [Frisch (1728), Kolenati (1859), Vitzthum (1932), Rudnick (1960)]. Kolenati (1859) et Vitzthum (1941) ont signalé les genres Meristaspis et Ancystropus sur les paupières et dans l'angle de l'œil de leurs hôtes; cette localisation n'a pas été retrouvée par Rudnick (1960). On relève, enfin, l'étonnant dermecos du genre Paraspinturnix vivant au niveau de l'orifice anal des chiroptères du genre Myotis en Amérique du Nord (Rudnick, 1960).

Les données relatives à la sex-ratio étaient jusqu'à présent restées quasi inexistantes : Tagiltzev (1971) a étudié un seul prélèvement. Haitlinger (1978) mentionne les nombres de mâles et de femelles capturés sans les analyser. En revanche, des observations plus précises concernent l'intensité de l'infestation et le nombre de chiroptères parasités dans les genres Periglischrus (Herrin, 1975), et Spinturnix (Haitlinger, 1978),

(4) Dermecos: «...microhabitat created by the host-skin and its outgrowths... » (Smit, 1972). 
L'exploitation de nos données recueillies durant les années 1977 à 1979 permet de définir le dermecos, la sex-ratio, l'intensité du parasitisme et le pourcentage de chiroptères parasités chez plusieurs espèces de Spinturnicidae tout au long de l'année.

\section{Spinturnix myoti (Kolenati, 1856)}

Spécificité : cette espèce est signalée par divers auteurs sur plusieurs hôtes dont les plus fréquents sont: Myotis myotis, Myotis bechsteini, Myotis capaccinii, Myotis oxygnathus.

En fait, $S$. myoti est un complexe dont nous avons déjà séparé Spinturnix andegavinus Deunff, 1977 parasite de Myotis daubentoni. Des études plus précises, à la fois morphologiques et éco-éthologiques permettront de mieux connaître la spécificité de S. myoti dont l'hôte-type est Myotis myotis myotis (in Rudnick, 1960). Avec Uchikawa et Wada (1979) nous considérons que la diagnose de ce Spinturnix doit être totalement révisée. Le problème du parasite de $M$. daubentoni ( $S$. andegavinus) est, quant à lui, résolu du fait de l'originalité des caractères morphologiques et surtout biologiques et écologiques de cette espèce ; toutefois, c'est l'hôte qui vient à son tour d'être scindé: Myotis daubentoni et $M$. nathalinae Tupinier, 1977. L'espèce S. daubentoni (Kolenati, 1857), exhumée par Haitlinger (1978) doit être considérée comme nomina nuda (Deunff, 1977).

D'autres espèces d'hôtes sont purement accidentelles, par exemple, Plecotus auritus, Rhinolophus ferrumequinum ou encore Miniopterus schreibersi. Quant aux captures de S. myoti sur M. mistacinus (sic) par Tagiltzev (1971), nous considérons qu'il s'agit d'une erreur d'identification, l'espèce étudiée étant probablement $S$. mystacinus.

\section{Structures des populations d'hôtes.}

(Notre étude écologique concerne ici uniquement Myotis myotis).

\section{Hiver.}

Pendant la saison froide, Myotis myotis mâles et femelles ont été trouvés dans les grottes, ou les cavités souterraines sensu lato, solitaires, par couples ou en essaims peu importants (3 à 20 individus environ), plusieurs essaims de ce type pouvant coexister dans une même station.

Eté.

Durant la période estivale, dans les combles ou greniers, nous avons trouvé presque exclusivement des femelles gestantes ou accompagnées de leurs jeunes. Les 
rassemblements sont alors plus importants et réunissent plusieurs centaines d'individus. La ségrégation des sexes est très nette.

\section{Dermecos.}

S. myoti se rencontre sur l'ensemble du patagium de son hôte. Mais, durant la période estivale on trouve quelques individus sur l'uropatagium du fait de la surpopulation ectoparasitaire (530 captures au total).

\section{Sex-ratio.}

Le calcul sur l'ensemble de l'année donne une sex-ratio de 0,54.

Durant la période estivale d'avril à octobre, elle est de 0,67 et pendant la période hivernale, de novembre à mars, ce chiffre descend à 0,43 .

Nous avons donc toujours une sex-ratio très en faveur des femelles, ce phénomène se retrouve à des degrés divers chez toutes les espèces étudiées.

Ces résultats sont nettement différents de ceux rapportés par Haitlinger (1978), les nombres des mâles et des femelles étant dans son travail à peu près équivalents : le petit nombre de parasites qu'il a étudiés explique peut-être cette discordance.

Notons encore que les $S$. myoti trouvés sur le guano (cf. infra), présentent une sex-ratio inversée $($ s.-r. $=1,36)$. Ceci peut s'expliquer, si la fécondation a lieu à cette époque de l'année, par des déplacements plus importants chez les mâles à la recherche des femelles, le risque de se détacher de l'hôte étant alors plus grand.

\section{Phénologie (fig. 2 à 4 ).}

A partir d'octobre, les Myotis myotis quittent les gîtes d'été pour regagner les grottes et, dès le mois de novembre, on assiste à une chute des effectifs de $S$. myoti bien que le nombre des hôtes parasités reste assez grand (55 à $60 \%$ ). Peu à peu l'intensité du parasitisme va diminuer et le nombre des hôtes parasités va décrô̂tre également durant $\mathrm{la}$ période hivernale, de novembre à mars. La stase adulte, avec des mâles et des femelles en petit nombre, constitue la "forme de résistance » hivernale chez les Spinturnicidae. Le nombre de chiroptères parasités devient inférieur à $40 \%$ généralement et l'intensité du parasitisme est très basse (5) (2 à 4 le plus souvent).

Dès le mois d'avril, des changements interviennent dans les populations de Spinturnicidae. Chez plusieurs espèces dont $S$. myoti, nous avons noté une diminution nette de l'intensité du parasitisme alors que le nombre d'hôtes parasités augmente. Ce phénomène est peut-être dû à la dissémination des parasites après l'hibernation des hôtes: les Spinturnicidae se répartiraient sur les hôtes disponibles avant

(5) L'intensité du parasitisme calculée par rapport au nombre d'hôtes parasités est nommée intensité relative (I.r.). 
la poussée démographique estivale. Un tel comportement erratique des adultes peut favoriser la rencontre des sexes.

En été, on assiste à une véritable explosion démographique chez $S$. myoti, avec 20 parasites par hôte en moyenne (souvent plus de 30). Les hôtes sont alors parasités à $100 \%$. Ce rythme de reproduction très intense est probablement une des causes de la présence de cette espèce dans le guano, du fait de la surpopulation des parasites.

Ce sont les quelques couples subsistant sur l'hôte en fin de saison estivale qui vont constituer les formes de résistance hivernales et permettre ainsi la survie de l'espèce. Le cycle de reproduction de $S$. myoti suit donc d'assez près celui de son hôte sans que l'on puisse actuellement préciser l'origine biotique ou abiotique de ce phénomène.

\section{Présence de $S$. myoti sur le guano.}

De nombreux auteurs ont fait mention de la présence d'ectoparasites de chiroptères sur le guano. Ainsi, par exemple, Jeannel (1926), a noté l'abondance de Nyctéribies et de Siphonaptères (6) sur ce substrat. Nous ne connaissons que deux références, par contre, concernant la présence de Spinturnicidae sur ce milieu : Holdenried et al. (1951) qui ont trouvé des Spinturnix sp. de Myotis yumanensis, libres sur le substrat ("... found free on the rosts at various times ") en Californie; Tagiltsev (1971) qui mentionne la présence de S. myoti (parasite de Myotis oxygnathus) sur le guano (... ectoparasites mainly ticks of Spinturnicidae streamed from above and actively crawled from below in large numbers... at the end of July...).

Durant le mois de juillet, nous avons constaté, à Durtal, l'abondance de $S$. myoti sur le guano, toutes les stases étaient représentées. La densité atteignait 140 individus pour $900 \mathrm{~cm}^{2}(30 \mathrm{~cm} \times 30 \mathrm{~cm})$, soit : 65 mâles, 50 femelles et 25 nymphes. Si l'on compare le nombre total de parasites pouvant vivre sur l'hôte et le nombre des Spinturnicidae présents sur le guano, on note un déséquilibre important en faveur des individus "libres ». En effet, la population de chiroptères présente à cette époque peut être estimée à 300 individus environ; si l'intensité moyenne du parasitisme atteint 20 par chauve-souris, cela fait 6000 Spinturnicidae. Or les acariens étaient présents sur le guano sur une surface d'environ $30 \mathrm{~m}^{2}$. Si l'on se limite à la surface directement située sous la colonie, soit un carré de $4 \mathrm{~m}$ sur $4 \mathrm{~m}\left(16 \mathrm{~m}^{2}\right)$, la population de $S$. myoti libre atteindrait déjà 25000 individus, ce qui est énorme, mais très inférieur à la réalité car les Spinturnicidae étaient présents, en fait, sur une aire beaucoup plus grande; le fait de poser la main sur le sol quelques secondes permettait de dénombrer plusieurs Spinturnicidae se déplaçant rapidement sur la peau.

(6) La présence des Nyctéribies comme celle des Spinturnicidae est accidentelle; celle des siphonaptères est normale, les Ischnopsyllidae qui sont ici concernés se développant à l'état larvaire dans ce milieu. 

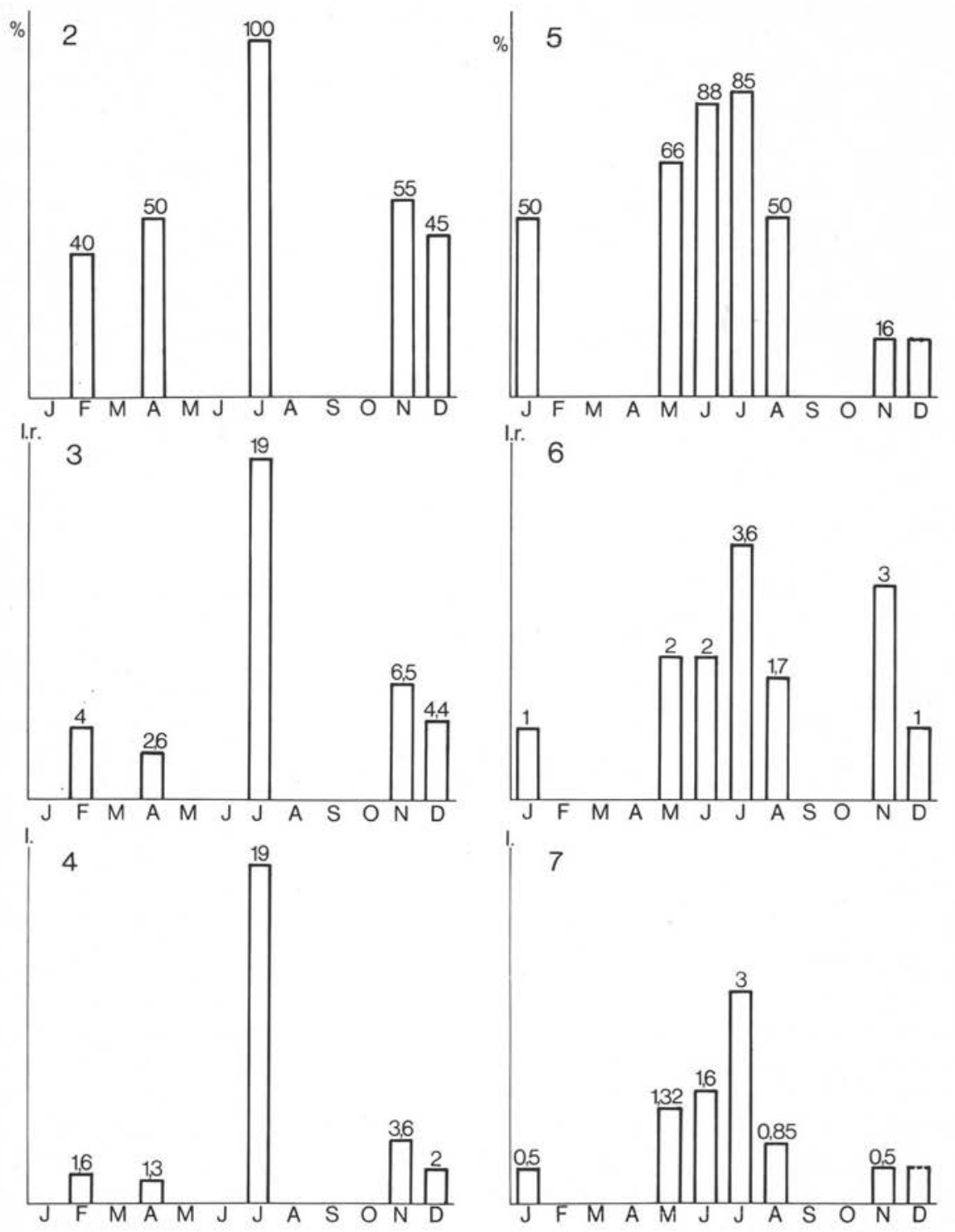

Planche I

Fig. 2. Variations annuelles du taux de parasitisme de M. myotis par S. myoti.

Fig. 3. Variations annuelles de l'intensité relative de l'infestation de M. myotis par S. myoti.

Fig. 4. Variations annuelles de l'intensité de l'infestation de M. myotis par S. myoti.

Fig. 5. Variations annuelles du taux de parasitisme de M. emarginatus par S. emarginatus.

Fig. 6. Variations annuelles de l'intensité relative du parasitisme de $M$. emarginatus par S. emarginatus.

Fig. 7. Variations annuelles de l'intensité du parasitisme de $M$. emarginatus par S. emarginatus. 
La présence de ces parasites dans le guano est difficilement explicable et nous n'avons noté ce fait que pour cette seule espèce. Plusieurs prélèvements de guano sous des colonies de Rhinolophes et de Myotis emarginatus se sont révélés négatifs.

En fait, la présence de ces ectoparasites sur le sol n'est pas obligatoirement sans issue, car lors de l'apprentissage du vol, beaucoup de jeunes chauves-souris tombent et rampent sur le guano où elles peuvent facilement se parasiter. Nous en avons d'ailleurs vu, fréquemment, tombés des essaims et rampant sur le sol, ou s'y posant au cours de leurs premiers vols; ils étaient porteurs à chaque fois, non seulement d'acariens très nombreux, mais aussi de siphonaptères et de cimicidés, ces deux derniers étant peu abondants sur les chauves-souris restées dans l'essaim. Il peut donc s'agir d'un mode de contamination classique.

L'abondance de $S$. myoti sur ce substrat ne dure, semble-t-il, que quelques semaines et est vraisemblablement dû à un surparasitisme des hôtes. On peut relever cependant que cette explosion démographique coïncide avec la mise bas des chauves-souris et on peut admettre l'existence d'un facteur hormonal (hormone sanguine ou phéromone) dans la reproduction des (ou au moins de ce) Spinturnix.

\section{Spinturnix emarginatus (Kolenati, 1856)}

\section{Spécificité.}

Le seul hôte connu jusqu’à présent pour cette espèce est Myotis emarginatus. Malgré un étroit contact interspécifique estival entre les colonies de $M$. emarginatus et de Rhinolophus ferrumequinum, le passage des parasites d'une espèce à l'autre est très rare et peut être considéré comme purement accidentel.

\section{Structure des populations d'hôtes.}

Hiver.

Durant la période hivernale nous n'avons trouvé que des individus solitaires dans les grottes et les cavités souterraines. Cette espèce se retire généralement dans les zones profondes, plus chaudes et plus stables, de l'abri ; elle choisit généralement d'assez larges fissures de la voûte, où elle pend sans contact avec les parois.

Eté.

A cette époque de l'année, nous n'avons étudié que des colonies constituées presque exclusivement de femelles gestantes et de jeunes dans les greniers. Les essaims sont alors très denses rassemblant plusieurs dizaines d'individus. Nous avons noté régulièrement l'association écologique entre $M$. emarginatus et $R$. ferrumequinum dans des essaims mixtes ou contigus. 


\section{Dermecos.}

La répartition de cette espèce sur son hôte est exclusivement limitée au patagium (152 captures).

\section{Sex-ratio.}

La s.-r. globale est de 0,71 avec un chiffre de 0,74 en été et de 0,25 seulement en hiver. La sex-ratio est donc en faveur des femelles, particulièrement en hiver où la mortalité des mâles semble importante. Toutefois à cette époque, le nombre de captures des hôtes est très faible de même que l'intensité du parasitisme, il faut donc considérer ce chiffre hivernal comme provisoire, une étude sur un échantillonnage plus important étant nécessaire.

\section{Phénologie (fig. 5 à 7).}

A partir de septembre, l'intensité du parasitisme décroît rapidement, mais le taux d'infestation reste élevé. En revanche, en décembre, on assiste à une chute brutale des effectifs de la population de $S$. emarginatus, ce phénomène se retrouvant chez les autres espèces de Spinturnicidae à maximum estival. Durant l'hiver, la population se maintient à un taux très bas, moins de $20 \%$ des hôtes étant parasités. L'intensité de l'infestation est minimale, le plus souvent on ne trouve qu'un Spinturnix par chauve-souris.

Au printemps, la population augmente peu à peu pour atteindre, en moyenne, 3 à 4 individus par hôte. Le maximum observé atteint 10 Spinturnix sur un même hôte. La faible intensité du parasitisme de cette espèce est probablement due à un rythme de reproduction relativement lent, ce qui est étonnant car les essaims estivaux de mise-bas sont apparemment, sur le plan de leur structure, tout aussi favorables au développement du parasite que ceux de $M$. myotis ou de $R$. ferrumequinum. Notons enfin que le cycle de reproduction suit à peu près celui des hôtes.

\section{Spinturnix mystacinus (Kolenati, 1857).}

\section{Spécificité.}

Nous étudions ici S. mystacinus sensu Rudnick (1960). Haitlinger (1978) a séparé deux sous-espèces $S$. mystacinus mystacinus et $S$. mystacinus brandti, mais le petit nombre de spécimens étudiés (6 de chaque sous-espèce) et une description insuffisante ne nous permettent pas de conclure quant à la validité de ces sous-espèces. Une étude plus approfondie est nécessaire. 
S. mystacinus sensu Rudnick (1960) est un parasite strict de Myotis mystacinus. Sa présence sur des hôtes différents (M. myotis, M. dasycneme et Plecotus auritus)' est purement accidentelle.

\section{Structure des populations d'hôtes.}

Nous n'avons capturé $M$. mystacinus que pendant la saison d'hiver suspendu aux parois des grottes et carrières souterraines, les gîtes d'été nous sont, en effet, inconnus. Les individus sont toujours solitaires, mais parfois assez nombreux dans certains abris.

Nous avons noté la présence de deux types morphologiques, différents par la taille comme par la coloration. Nous n'avons pas voulu les sacrifier et, par conséquent, le statut taxonomique de ces «types» ne peut être précisé. Les deux sont porteurs de ce Spinturnix.

\section{Dermecos.} tures).

La localisation des captures s'est limitée exclusivement au patagium (48 cap-

\section{Sex-ratio.}

La sex-ratio hivernale est de 0,6 , donc en faveur des femelles.

Phénologie. (fig. 8 à 10).

Nos prélèvements portent malheureusement sur la seule moitié hivernale de l'année. Nous ne pouvons que constater la stabilité de la population à cette saison. De novembre à février, l'effectif est presque constant et on ne retrouve pas la rupture d'effectif entre novembre et décembre comme chez les autres Spinturnix parasites de vespertilions.

\section{Eyndbovenia euryalis (Canestrini, 1884)}

\section{Spécificité.}

E. euryalis sensu Rudnick (1960) est un parasite régulier des Rhinolophes, mais Rhinolophus euryale en est l'hôte principal. Actuellement, des études systématiques plus complètes tendent à séparer ce taxon en plusieurs sous-espèces (Uchikawa et Dusbabek, 1978). La présence, signalée par plusieurs auteurs, de ce parasite sur Myotis myotis, M. emarginatus et Miniopterus schreibersi s'explique par une cohabitation de ces hôtes permettant un échange d'ectoparasites. 

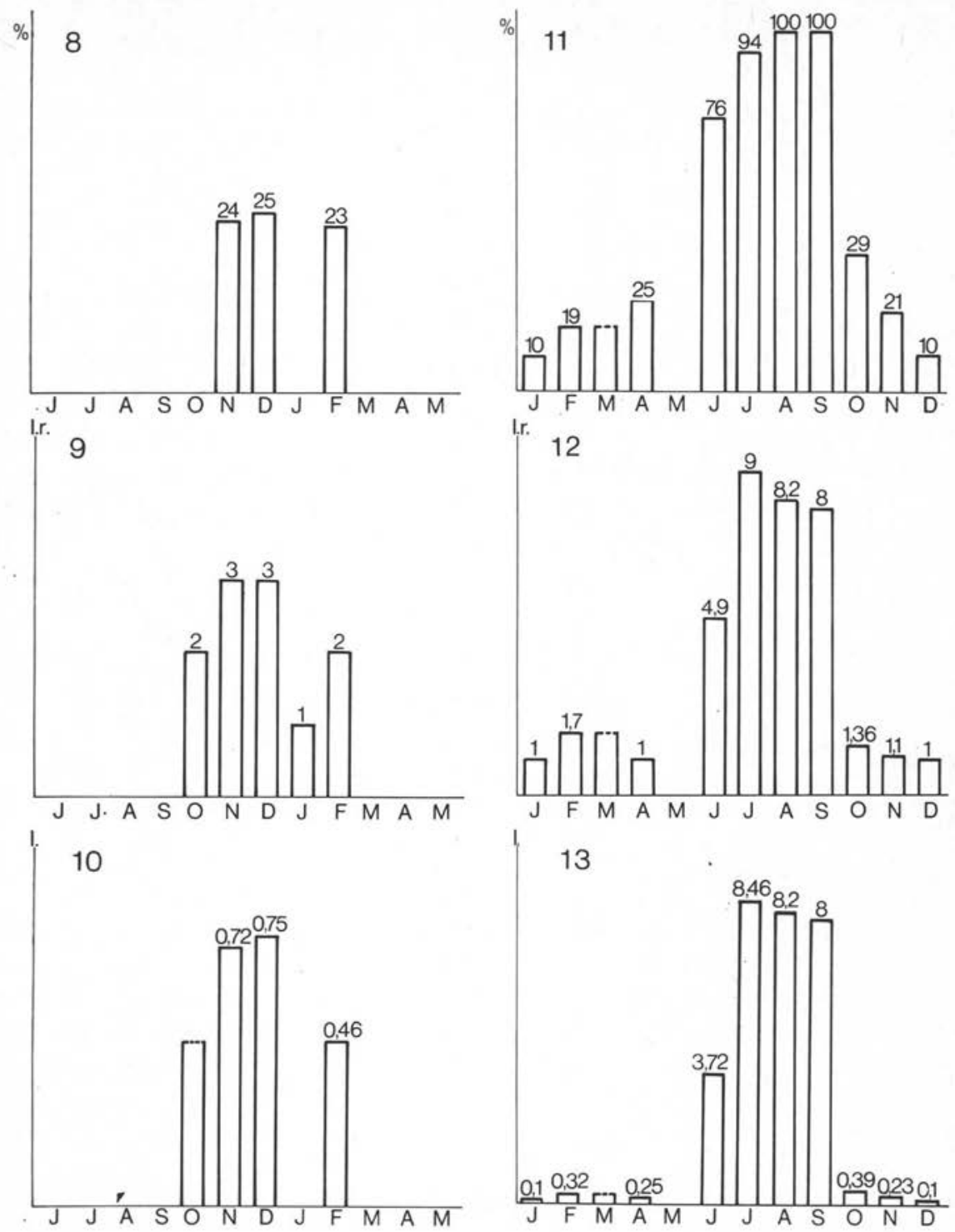

Planche II

Fig. 8. Variations annuelles du taux de parasitisme de M. mystacinus par S. mystacinus.

Fig. 9. Variations annuelles de l'intensité relative du parasitisme de M. mystacinus par S. mystacinus.

Fig. 10. Variations annuelles de l'intensité du parasitisme de M. mystacinus par S. mystacinus.

Fig. 11. Variations annuelles du taux de parasitisme de $R$. ferrumequinum par E. euryalis.

Fig. 12. Variations annuelles de l'intensité relative de l'infestation de $R$. ferrumequinum par E. euryalis.

Fig. 13. Variations annuelles de l'intensité de l'infestation de $R$. ferrumequinum par E. euryalis. 


\section{Structure des populations d'hôtes.}

Nous n'avons étudié que les, ,arasites de Rhinolophus ferrumequinum, nos captures de $R$. euryale étant trop peu nombreuses.

Hiver.

Pendant l'hiver, mâles et femelles ont été retrouvés dans les grottes et les cavités souterraines. Dans un même abri cohabitent des rhinolophes solitaires ou des «foules » dont l'importance varie de quelques individus à plusieurs centaines de chaives-souris, formant, dans ce dernier cas, de véritables essaims, de structure lâche, de mâles et de femelles.

Eté.

Les colonies estivales situées sous la toiture des combles ou des greniers sont presque exclusivement constituées de femelles et de jeunes. Certains abris rassemblent plusieurs centaines d'individus dans les classiques colonies de mise-bas. On rencontre fréquemment l'association écologique entre $R$. ferrumequinum et $M$. emarginatus (Brosset, 1966).

\section{Dermecos.}

Pendant l'été, on peut considérer que cette espèce a comme dermecos presque exclusif les membranes alaires (400 captures). La surpopulation estivale sur le patagium ou les manipulations de l'hôte par l'observateur peuvent expliquer les quelques captures faites sur l'uropatagium (30 captures).

En revanche, durant la saison hivernale, E. euryalis ne se rencontre plus sur le patagium en dépit du grand nombre d'hôtes étudiés. L'hypothèse d'une disparition totale de l'espèce sur l'hôte pendant la saison d'hiver n'étant pas envisageable puisqu'il s'agit d'ectoparasites obligatoires, nous avons recherché cette espèce dans le pelage, bien que théoriquement ces acariens soient confinés aux membranes. Nous $\mathrm{y}$ avons effectivement retrouvé $E$. euryalis. On rencontre cette espèce dans une région du pelage (dorsale le plus souvent) située en bordure du patagium et sur une longueur de 1 à $1,5 \mathrm{~cm}$ au-dessus du fémur. Les acariens sont fixés sur la peau entre les poils bordant cette membrane. Ils y sont toutefois en petit nombre.

\section{Sex-ratio.}

Durant la période estivale, la s.-r. $=0,55$.

Pendant l'hiver, saison défavorable pour cette espèce, il semble que la mortalité soit plus importante chez les femelles que chez les mâles : la s.-r. $=0,64$ sur la période allant de novembre à mars. 
Phénologie (fig. 11 à 13).

E. euryalis est encore une espèce à reproduction estivale. Le pic de population se situe en juillet avec, en moyenne, 9 individus par hôte parasité (maximum observé $=20$ ). Ce parasitisme intense persiste de juillet à septembre inclus avec un taux de parasitisme élevé variant de $94 \%$ en juillet à $100 \%$ en août et septembre chez $R$. ferrumequinum. A partir d'octobre, avec le retour des chiroptères dans les abris souterrains, la chute de population est considérable ( 1,36 individu par hôte parasité). Le taux de parasitisme (29\% en octobre) suit la même évolution. Cette variation de l'effectif d'ectoparasites, très brutale entre septembre et octobre, va évoluer plus lentement en novembre, puis en décembre où elle atteint son minimum, le taux de parasitisme étant alors aux environs de 10 à $20 \%$. Quelques adultes vont alors survivre, dans le pelage, en bordure de patagium, en attendant la période estivale favorable. Dès le printemps, on assiste à une lente remontée des effectifs, cette augmentation de population est nette en juin. En quelques semaines l'intensité du parasitisme passe de moins de 2 individus à 5 en moyenne par hôte parasité : le taux de parasitisme évoluant de $20 \%$ en avril à $76 \%$ en juin, puis $94 \%$ en juillet. Comme chez $S$. myoti, on observe donc une véritable explosion démographique estivale due à une fécondité élevée et à une durée très brève du développement embryonnaire.

\section{Paraperiglischrus rbinolopbinus (Koch, 1841)}

\section{Spécificité.}

P. rhinolophinus est un parasite des Rhinolophidae. La présente étude concerne exclusivement les parasites de Rhinolophus ferrumequinum.

Strueture des populations d'hôtes (cf. E. euryalis).

\section{Dermecos.}

Nous avons noté, en ce qui concerne cette espèce, une localisation différente pour les mâles, d'une part, les femelles et les nymphes, d'autre part. Les mâles n'ont été observés que sur le patagium (56 captures), hiver comme été. En revanche, en hiver, femelles et nymphes sont localisées sur l'uropatagium dorsal (380 captures); durant l'été, nous avons trouvé quelques femelles sur le patagium (10 captures), l'essentiel de la faible population estivale de femelles restant situé sur l'uropatagium.

Il faut rappeler, à ce sujet, que $P$. rhinolophinus, parasite des Rhinolophidae, en hibernation, a la particularité d'avoir un uropatagium replié, dorsalement, contrairement aux autres chiroptères de nos régions. Les parasites fixés générale- 

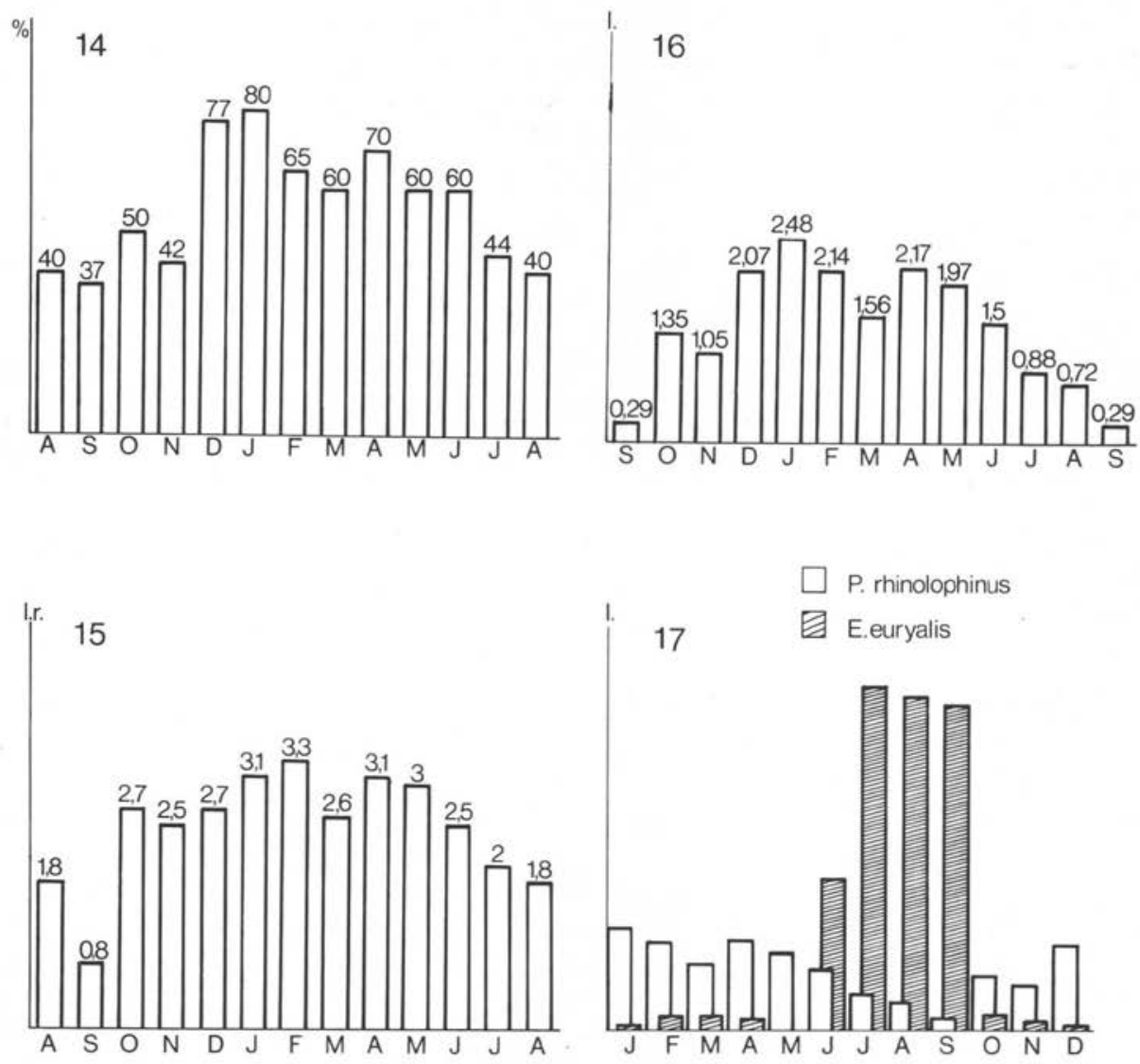

Planche III

Fig. 14. Variations annuelles du taux de parasitisme de $R$. ferrumequinum par P. rhinolophinus.

Fig. 15. Variations annuelles de l'intensité relative de l'infestation de $R$. ferrumequinum par P. rhinolophinus.

Fig. 16. Variations annuelles de l'intensité de l'infestation de $R$. ferrumequinum par P. rhinolophinus.

Fig. 17. Variations annuelles de l'intensité comparée de l'infestation de $R$. ferrumequinum par $P$. rhinolophinus et E. euryalis. 
ment le long de l'appendice caudal dans sa partie médiane sont donc placés dans la région profonde du repli de l'uropatagium. A ce niveau, la vascularisation est importante et peut favoriser le développement de $P$. rhinolophinus. Ne disposant pas de microsonde, nous n'avons pu mesurer les caractéristiques microclimatiques à l'intérieur du repli caudal. Toutefois, la face interne (ou dorsale), de cette membrane a toujours été trouvée très humide durant l'hivernage.

\section{Sex-ratio.}

Globalement, sur l'ensemble de l'année, la population est constituée à $87 \%$ de femelles (s.-r. $=0,15$ ). La sex-ratio est donc nettement en leur faveur. Mais, si l'on dissocie les effectifs estivaux et hivernaux, les résultats sont plus précis. La période estivale, d'avril à octobre, montre une sex-ratio de 0,49 alors que l'hiver, de novembre à mars, la s.r. $=0,08$. En été, le nombre de mâles est donc proportionnellement plus élevé que l'hiver (en juillet, la s.-r. atteint 0,8 ), alors que, comme nous le verrons, la population globale a son effectif minimum à cette époque.

Phénologie (fig. 14 à 16).

Pendant toute la saison estivale, période d'intense reproduction des autres espèces étudiées, nous avons relevé, chez $P$. rhinolophinus, un effectif très faible, avec un minimum en septembre, le nombre d'hôtes parasités étant lui-même très réduit. Devant ce résultat nous avons cherché, sans succès, d'autres localisations éventuelles de cette espèce dans le pelage et autour de l'anus. En effet, l'hiver, la majorité de la population se trouve dans le repli de l'uropatagium dorsal très humide, or, l'été, les membranes le sont beaucoup moins.

En rapprochant deux de nos observations précédentes : la variation de la sex-ratio de 0,08 l'hiver, à 0,8 en juillet, rééquilibrant partiellement les proportions de mâles et de femelles et la découverte de quelques femelles sur le patagium durant l'été, nous pensons qu'il est possible que ces deux faits favorisent la rencontre des sexes et que la fécondation ait lieu à cette période de l'année. Mais, actuellement, nos connaissances de la physiologie de la reproduction des Spinturnicidae sont encore trop incomplètes pour conclure sur ce point.

A partir du mois d'octobre on observe une lente remontée des effectifs avec un maximum de population en février. La reproduction de cette espèce a donc lieu l'hiver. Toutefois, l'intensité du parasitisme atteint seulement en moyenne 3 à 4 individus par hôte parasité, maximum : 13 , ce qui est très modeste comparativement à d'autres espèces comme $S$. myoti par exemple, où l'on assiste à une véritable « explosion" démographique pendant la période de reproduction. Cette fécondité réduite est connue chez les animaux vivant en régions froides et à reproduction hivernale et chez les Troglobies. 


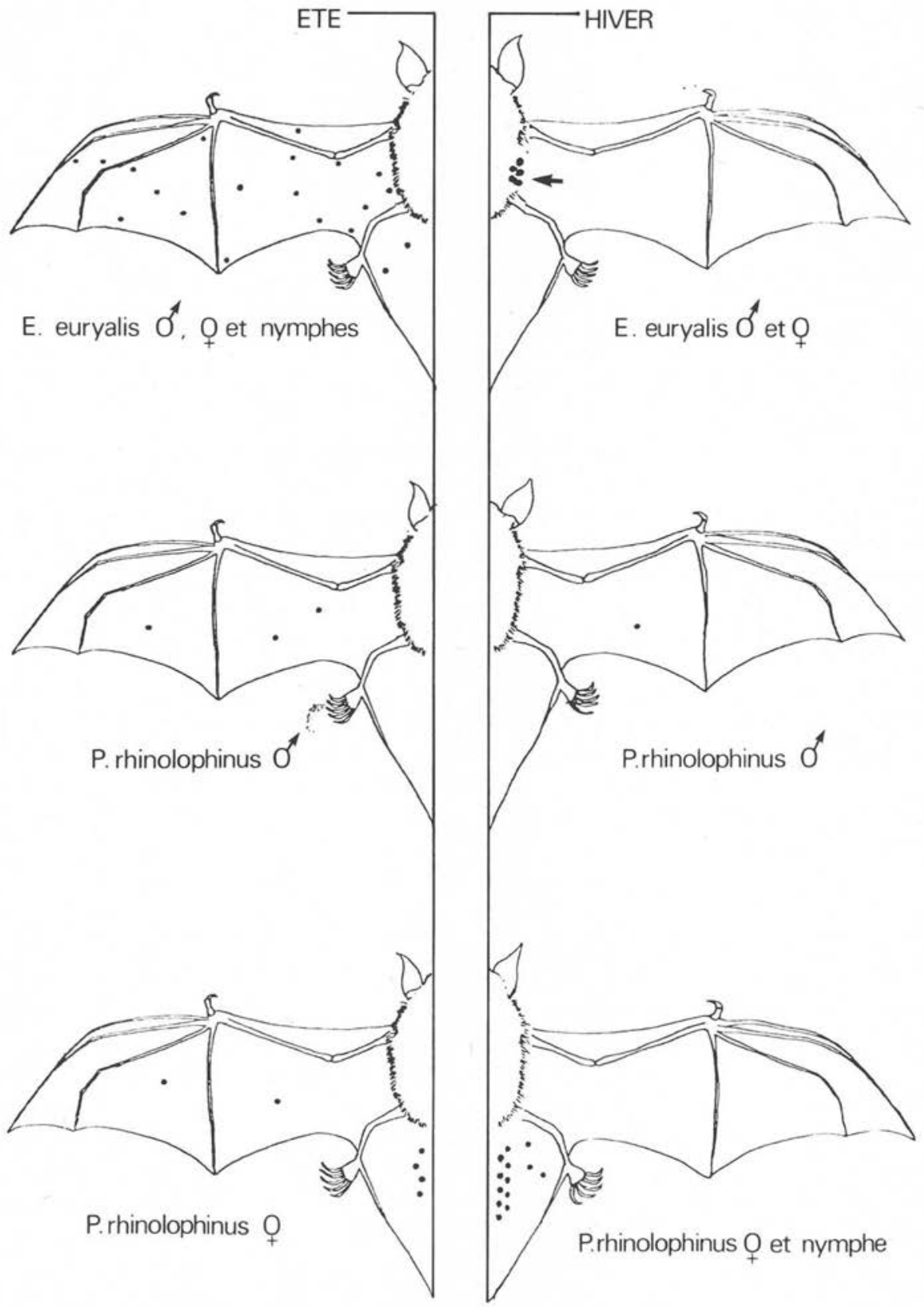

Fig. 18. Dermecos de E. euryalis et $P$. rhinolophinus sur $R$. ferrumequinum en été et en hiver (le nombre de points est proportionnel à l'intensité du parasitisme). 


\section{Discussion.}

Les observations précédentes, concernant la phénologie des Spinturnicidae, permettent de séparer les espèces étudiées en trois groupes:

- tout d'abord, les espèces du genre Spinturnix dont le dermecos est exclusivement le patagium (7) et qui ont une reproduction estivale avec une fécondité élevée le plus souvent,

- puis, le genre Eyndhovenia (E. euryalis), dont le dermecos suit des variations saisonnières, membranes alaires l'été et pelage l'hiver. La reproduction est également estivale, la fécondité élevée, mais la chute de population en début de saison froide est très brutale et est en faveur de l'hypothèse d'une "capture " par $R$. ferrumequinum du parasite de $R$. euryale qui est plus méridional, E. euryale ayant conservé ses exigences écologiques et en premier lieu, probablement, un thermopreferendum élevé,

- enfin, dans le genre Paraperiglischrus, le dermecos est très particulier, uropatagium pour les femelles et les nymphes et patagium pour les mâles. La phénologie est plus étonnante encore avec une reproduction hivernale, une relativement basse fécondité et un cycle de reproduction inverse de celui de l'hôte. Les Spinturnicidae sont des parasites très anciens, très spécialisés et les adaptations morphologiques sont nombreuses, ce qui rend difficile l'étude de leur phylogénie (Fain, 1978, in litteris). Celle-ci ne peut donc nous aider à mieux comprendre ces phénomènes.

L'originalité de ces observations réside dans la découverte d'un véritable balancement des populations de Spinturnicidae chez le grand rhinolophe $R$. ferrumequinum entre E. euryalis l'été, et $P$. rhinolophinus l'hiver. Les deux populations de parasites se succèdent dans le temps sans jamais entrer en compétition, d'autant plus que leur localisation sur l'hôte est différente (patagium pour E. euryalis et uropatagium pour P. rhinolophinus) (fig. 17 et 18).

Dans la plupart des espèces étudiées nous avons noté, avant la période de reproduction, une baisse sensible de l'intensité moyenne d'infestation, alors que le taux d'infestation augmente (par exemple, $S$. myoti). Ce phénomène peut s'expliquer par une dissémination de ces acariens précédant l'augmentation globale de la population, cet erratisme favorisant peut-être la rencontre des sexes. Pendant la saison défavorable, les «stases de résistance " observées sont les adultes dont quelques individus survivent jusqu'à la belle saison et permettent, ainsi, la persistance de l'espèce.

Nous n'avons pu véritablement comparer le parasitisme en fonction du sexe des hôtes car, le plus souvent, nous ne retrouvons les mâles et les femelles hôtes que durant l'hiver; l'été, nous n'observons plus que les colonies de mise-bas avec les jeunes et les femelles. Toutefois, en ce qui concerne la transmission des parasites d'hôte à hôte, le phénomène est évident chez les jeunes en contact avec les femelles

(7) Les parasites de $M$. daubentoni et $P$. austriacus ont été récoltés uniquement sur le patagium, le petit nombre des captures ne nous permet pas actuellement de présenter la phénologie de ces espèces. 
l'été. Chez les mâles, il est probable que la transmission ou les réinfestations se passent en automne lors de l'accouplement, ou l'hiver dans les essaims mixtes.

Ces variations de population peuvent vraisemblablement subir des modifications sensibles en fonction des conditions climatiques, ces facteurs abiotiques agissant sur les chiroptères et sur leurs ectoparasites.

\section{Conclusion.}

Nous avons rassemblé nos premières observations écologiques sur les Spinturnicidae. Celles-ci sont encore très fragmentaires, mais les difficultés d'observation de ces parasites dans leur milieu naturel sont considérables. Nous souhaiterions, peu à peu, préciser nos résultats et étendre notre étude à d'autres espèces. Par la suite, des comparaisons avec l'évolution et le comportement des espèces tropicales seraient d'un grand intérêt, en particulier pour les parasites de rhinolophes, chauves-souris originaires des zones chaudes. Mais, en préalable, à des comparaisons écologiques entre les genres et les espèces des différentes parties du monde, il est nécessaire d'entreprendre une révision de l'ensemble de la systématique de la famille. Un tel travail n'a pas été fait depuis vingt ans (Rudnick, 1960) et devient indispensable en raison du nombre d'espèces décrites depuis cette date.

\section{Bibliographie}

Belashova V.S.: Méthodes d'immobilisation et d'entretien des chauves-souris au laboratoire (en russe). Akad.-Nauk Kazakh. S.S.R., Trudy Inst. Zool., 1964, 22, 205-206.

Brosset A. : La biologie des Chiroptères. Masson, Paris, 1966, 240 p.

Davis W. H., Luckens M. M.: Use of big brown bats (Eptesicus fuscus) in biomedical research. Lab. Animal Care U.S.A., 1966, 16, 224-227.

Deunff J.: Observations sur les Spinturnicidae de la région paléarctique occidentale (Acarina, Mesostigmata). Spécificité, répartition et morphologie. Acarologia, 1977, 18, 602-617.

Dusbabek F.: The zone of bat acarinia in central Europe. Folia Parasitologica, 1972, 19, 139-154.

Fraysse J., Fraysse C.: Les troglodytes en Anjou à travers les âges, 1963, 165 p.

Frisch J. L.: Beschreibung von allerlei Insecten in Deuschland, nebst nuetzlichen Anmerkungen und noethigen Abbildungen von diesem kriechenden und fliegenden Inlaendischen Gewuerme, zur Bestaetigung und Fortsetzung der gruendlichen Entdeckung, so einige von der Natur dieser Creaturen heraus gegeben, und zur Ergaentzung und Verbesserung der andern. Cristoph Gottlieb Nicolai, Berlin, 1728, vol. 7, 31 p., 22 pl.

Grasse P.P.: Traité de Zoologie : Mammifères, 17, fasc. 2, Masson, Paris, 1955, pp. 1173-2300.

Haitlinger R.: External parasites of lower Silesian bats. III. Spinturnicidae, Argasidae, Ixodidae (Acarina). Wiad. Parazytol., 1978, 24, 476-490.

Herrin C.S.: Spinturnicid mites of Venezuela (Acarina: Spinturnicidae). Brigham Young Univ. Sci. Bull., 1975, 20, 1-71.

Holdendried R., Evans F. C., Longanecker D.S. : Host-parasite-disease relationships in a mammalian community in the central coast range of California. Ecol. Monogr., 1951, 21, 1-18. 
Jeannel R.: Faune cavernicole de la France avec une étude des conditions d'existence dans le domaine souterrain. Paul Lechevalier, Paris, 1926, 334 p.

Kolenati F.A.: Synopsis prodroma der Flughaut-Milben (Pteroptida) der Fledermäuse. Wien. Ent. Monatschr., 1857, 1, 59-61.

Kolenati F. A.: Beiträge zur Kentniss der Arachniden. Sitzungsb. K. Akad. Wissensch. Wien, Math.Naturw. Cl., 1859, 33, 69-89.

Reisen W.K., Kennedy M. L., Reisen N.T.: Winter ecology of ectoparasites collected from hibernating Myotis velifer (Allen) in southwestern Oklahoma (Chiroptera: Vespertilionidae). J. Parasitol., 1976, 62, 628-635.

Rothschild M., Ford B. : Factors influencing the breeding of the rabbit flea (Spilopsyllus cuniculi) : A spring-time accelerator and a kairomone in nestling rabbit urine with notes on Cediopsylla simplex, another " hormone bound " species. J. Zool. London, 1973, 170, 87-137.

Rudnick A.: A revision of the mites of the family spinturnicidae (Acarina). Univ. Calif. Publ. Ent., $1960,17,157-284$.

Smit F.G. A. M.: On some adaptative structures in siphonaptera. Folia Parasitol., 1972, 19, 5-17.

Tagiltzev A. A.: Arthropods collected from Myotis mistacinus Kohl. and M. oxygnathus Mont. in Zaysan Hollow. Parasitology, 1971, 5, 434-436.

Tesh R. B., Arata A. A. : Bats as laboratory animals. Health Lab. Sci., 1967, 4, 106-112.

Tupinier Y.: Description d'une chauve-souris nouvelle: Myotis nathalinae nov. sp. (Chiroptera, Vespertilionidae). Mammalia, 1977, 41, 327-340.

Uchikawa K., Dusbabek F.: Studies on Mesostigmatid Mites Parasitic on Mammals and Birds in Japan. VIII. Bat Mites of the genus Eyndhovenia Rudnick, 1960, with Redescription of Eyndhovenia euryalis euryalis (Canestrini, 1884). Bull. Nat. Sci. Mus., Tokyo, 1978, 4, 245-261.

Uchikawa K., Wada Y.: Studies on mesostigmatid mites parasitic on mammals and birds in Japan. IX. Bat mites of the genus Spinturnix von Heyden 1829 (Part I) (Spinturnicidae). Jap. J. Sanit. Zool., 1979, 30, 121-125.

Vitzthum G.H. : Neue parasitische Fledermausmilben aus Venezuela. Z. Parasitenk., 1932, 4, 1-47.

Vitzthum G.H.: Acarina in Bronn's Klassen und Ordnungen des Tierreiches, Band 5, IV Abteilung ; Arachnoidea and Kleinere ihnen nahegestellte Gruppen. 5. Buch: Acarina. Leipzig; Becker und Erler, 1941, 4 Lieferung, 481-640, fig. 429-448. - 5 Lieferung, 641-800, fig. 449-486.

Wimsatt W. A. : Biology of Bats. Vol. II, Academic Press, New York, 1970, 477 p. 